\title{
Le devenir des femmes en chirurgie
}

La diversité des mondes de socialisation

Women and surgery: diversity in the spheres of socialization

\section{Régine Bercot}

\section{(2) OpenEdition}

\section{Journals}

Édition électronique

URL : https://journals.openedition.org/travailemploi/6563

DOI : 10.4000/travailemploi.6563

ISSN : 1775-416X

Éditeur

DARES - Ministère du Travail

Édition imprimée

Date de publication : 1 janvier 2015

Pagination : $37-52$

ISSN : 0224-4365

Référence électronique

Régine Bercot, «Le devenir des femmes en chirurgie », Travail et Emploi [En ligne], 141 | janvier-mars 2015, mis en ligne le 01 janvier 2017, consulté le 03 juillet 2022. URL : http://journals.openedition.org/ travailemploi/6563 ; DOI : https://doi.org/10.4000/travailemploi.6563 


\title{
Le devenir des femmes en chirurgie : la diversité des mondes de socialisation $\left.{ }^{*}\right)$
}

\author{
Régine Bercot ${ }^{(*)}$
}

Comment expliquer que la chirurgie soit si peu attractive pour les femmes? Dans quelle mesure leur absence est-elle rédhibitoire? Les femmes de la génération actuelle entretiennent un rapport au travail très différent de leurs aînées. Majoritaires au concours d'entrée en médecine, elles sont peu attirées par la chirurgie, en tous les cas par certaines spécialités chirurgicales. On peut de fait y distinguer deux "mondes sociaux", se caractérisant par deux ethos différenciés dans lesquels les femmes s'insèrent puis font carrière. Le premier monde, celui de la chirurgie traditionnelle, est incontournable car il est au centre de la formation. D'autres spécialités développent, quant à elles, un ethos alternatif plus favorable à l'entrée des femmes, auxquelles elles ne peuvent toutefois accéder que par la suite. Étant donné les modalités d'un apprentissage effectué sur le mode du compagnonnage, l'accueil fait aux étudiants (externes et internes) et les interactions avec les chirurgiens seniors jouent un rôle essentiel sur l'envie de devenir chirurgien-ne. Nous verrons que les femmes doivent négocier leur identité sociale durant la formation pour se ménager l'aide des seniors et pouvoir supporter ce passage en chirurgie traditionnelle.

L'arrivée des femmes dans les études de médecine a été spectaculaire. Rappelons pourtant que les études de médecine, à l'origine interdites aux femmes, ne s'ouvriront à elles que très progressivement ${ }^{(1)}$. Leur nombre est si faible jusque dans les années 1950 qu'elles ne représentent pas la moindre concurrence pour les hommes. D'après Anne-Chantal HARDY (2005, p. 35), «après un premier élan à la faveur de la première guerre mondiale, mais surtout au lendemain de la seconde, la féminisation des études médicales s'est opérée dans un contexte d'explosion de la démographie médicale, jusque dans les années soixante-dix». L'instauration du numerus clausus en 1971 stabilise le nombre d'étudiants admis en études de médecine alors que, dans le même temps, non seulement la proportion de femmes réussissant le concours ne cesse de progresser mais leur rang de classement les place parmi les premières aux épreuves classantes nationales $(\mathrm{ECN})$. L'accès des femmes à ces parcours de prestige constitue un vrai changement social.

Cependant, cette entrée massive des femmes dans les professions médicales se traduit par une division sexuée des spécialités, toujours visible aujourd'hui, comme l'atteste le tableau suivant.

(*) Je remercie chaleureusement Madame Véronique Suhard de l'Irdes (Institut de recherche et documentation en économie de la santé) qui m'a aidée à retrouver des sources statistiques. (**) Université Paris-8, Cresppa-GTM (Centre de recherches sociologiques et politiques de Paris - Genre, travail, mobilités); EHESP (École des hautes études en santé publique), MOS (Management des organisations de santé); reginebercot@ gmail.com

(1) En 1902, les femmes représentent $5 \%$ de la profession (HARDY, 2005, p. 36).
Tableau 1 : Proportion de femmes dans les différentes chirurgies et dans quelques spécialités non chirurgicales, 1 er janvier 2014

\begin{tabular}{|l|r|c|}
\hline \multicolumn{1}{|c|}{ Spécialités } & $\begin{array}{r}\text { Total } \\
\text { France } \\
\text { entière }\end{array}$ & $\begin{array}{c}\text { Part des } \\
\text { femmes } \\
\text { (\%) }\end{array}$ \\
\hline Anesthésie-réanimation & 10949 & 35,5 \\
Cardiologie et maladies vasculaires & 6796 & 23,2 \\
Dermatologie et vénéréologie & 4076 & 68,0 \\
Endocrinologie et métabolisme & 1778 & 73,6 \\
Gastro-entérologie et hépatologie & 3570 & 29,8 \\
Génétique médicale & 233 & 70,4 \\
Gériatrie & 1537 & 57,2 \\
Gynécologie médicale & 3476 & 71,5 \\
Neurologie & 2289 & 46,0 \\
Pédiatrie & 7710 & 66,1 \\
Radio-diagnostic et imagerie médicale & 8467 & 33,3 \\
Psychiatrie & 14831 & 48,5 \\
Médecine du travail & 5694 & 70,4 \\
Santé publique et médecine sociale & 1666 & 59,4 \\
Médecine générale & 102140 & 42,4 \\
Chirurgie, dont & $\mathbf{2 5 8 0 2}$ & $\mathbf{2 6 , 1}$ \\
\hline Chirurgie générale & 3630 & 15,8 \\
Chirurgie infantile & 287 & 39,7 \\
Chirurgie orthopédique et & 3057 & 4,4 \\
traumatologie & 929 & 13,6 \\
Chirurgie viscérale et digestive & 4381 & 43,8 \\
Gynécologie et obstétrique & 525 & 12,8 \\
Neurochirurgie & 5855 & 44,0 \\
Ophtalmologie & 3070 & 22,1 \\
ORL et chirurgie cervico-faciale & & \\
\hline
\end{tabular}

Champ : Ensemble des médecins en activité dans certaines spécialités, dont la chirurgie (France métropolitaine et départements d'outremer). Source : «Effectifs des médecins par spécialité et mode d'exercice au

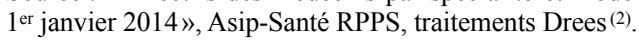

(2) Asip-Santé : Agence des systèmes d'information partagés de santé;

RPPS : Répertoire partagé des professionnels de santé;

Drees : Direction de la recherche, des études, de l'évaluation et des statistiques. 
Pour ce qui est de la chirurgie en particulier, l'écart hommes-femmes reste important mais tend à diminuer au fil du temps puisque la proportion de femmes augmente peu à peu, de manière régulière (voir tableau 2).

Tableau 2 : Évolution de la répartition par sexe des chirurgiens entre 1984 et 2010

\begin{tabular}{|c|c|c|c|c|c|c|}
\hline & $\mathbf{1 9 8 4}$ & $\mathbf{1 9 8 9}$ & $\mathbf{1 9 9 5}$ & $\mathbf{2 0 0 0}$ & $\mathbf{2 0 0 5}$ & $\mathbf{2 0 1 0}$ \\
\hline Hommes & 6195 & 5979 & 5626 & 5843 & 5760 & 5400 \\
$(\%)$ & $(98,6)$ & $(97,7)$ & $(95,2)$ & $(93,7)$ & $(91,3)$ & $(88,5)$ \\
\hline Femmes & 91 & 140 & 281 & 393 & 549 & 703 \\
$(\%)$ & $(1,4)$ & $(2,3)$ & $(4,8)$ & $(6,3)$ & $(8,7)$ & $(11,5)$ \\
\hline Total & 6286 & 6119 & 5907 & 6236 & 6309 & 6103 \\
\hline
\end{tabular}

Champ: Ensemble des chirurgiens, France métropolitaine entre 1984 et 2010 .

Source: Asip-Santé, répertoire Adeli (Automatisation des listes), traitements Drees.

En chirurgie, la proportion des femmes est très inégale en fonction des spécialités. Elle est très faible en chirurgie orthopédique, urologique, vasculaire, ou viscérale et digestive. Elle est en revanche beaucoup plus importante en chirurgie infantile et gynécologie-obstétrique, où elle a sensiblement progressé au cours des dix dernières années, en ophtalmologie, où elle est restée stable (voir tableau 3).

Tableau 3 : Taux de féminisation par discipline chirurgicale

\begin{tabular}{|l|c|c|}
\hline \multicolumn{1}{|c|}{ Disciplines chirurgicales } & $\begin{array}{c}\text { Proportion de } \\
\text { femmes (en \%) } \\
\mathbf{1}^{\text {er janvier }} \\
\mathbf{2 0 0 5}\end{array}$ & $\begin{array}{c}\text { Proportion de } \\
\text { femmes en \% } \\
\mathbf{1}^{\text {er janvier }}\end{array}$ \\
\hline Chirurgie générale & 6,6 & 15,8 \\
\hline Chirurgie infantile & 32,6 & 39,7 \\
\hline $\begin{array}{l}\text { Chirurgie maxillo-faciale (et } \\
\text { stomatologie en 2014) }\end{array}$ & 27,8 & 18,2 \\
\hline $\begin{array}{l}\text { Chirurgie orthopédique et } \\
\text { traumatologique }\end{array}$ & 2,7 & 4,4 \\
\hline $\begin{array}{l}\text { Chirurgie plastique et recons- } \\
\text { tructrice (et esthétique en 2014) }\end{array}$ & 18,5 & 22,6 \\
\hline $\begin{array}{l}\text { Chirurgie thoracique et } \\
\text { cardiaque }\end{array}$ & 6,9 & 9,7 \\
\hline Chirurgie urologique & 2,1 & 4,4 \\
\hline Chirurgie vasculaire & 5,1 & 8,4 \\
\hline Chirurgie viscérale et digestive & 12,3 & 13,6 \\
\hline Gynécologie obstétrique & 36,2 & 43,8 \\
\hline Neurochirurgie & 8,9 & 12,8 \\
\hline Ophtalmologie & 43,3 & 44,0 \\
\hline Otorhinolaryngologie & 16 & 22,1 \\
\hline
\end{tabular}

Champ: Chirurgiens en activité selon la spécialité chirurgicale (France métropolitaine), 2005 -2014.

Sources : SicART D. (2005), Les médecins. Estimations au $1^{e r}$ janvier 2005, Document de travail, série «Statistiques», ${ }^{\circ}{ }^{8} 8$, Paris, Drees, p. 25 et «Effectifs des médecins par spécialité et mode d'exercice au 1 er janvier 2014», Asip-Santé RPPS, traitements Drees.

Tout en nous donnant des indications précieuses sur la place des femmes dans les différentes spécialités, ces chiffres ne nous disent rien sur la manière dont les étudiants effectuent leurs choix à l'issue des ECN. Les lignes de partage sont-elles les mêmes que dans celles des effectifs?
En 2012, un premier groupe de spécialités est classé en tête par les étudiants : il s'agit de spécialités dites médicales, s'exerçant d'ordinaire en libéral et permettant d'atteindre un haut niveau de revenus. $\mathrm{La}$ radio-diagnostic, la cardiologie, la dermatologie, la gastro-entérologie, la néphrologie et la médecine interne en font partie, tout comme deux spécialités chirurgicales, l'ophtalmologie et l'otorhinolaryngologie. En deuxième position suivent des spécialités exercées dans un cadre le plus souvent salarié, associées à une charge de travail importante et à des gardes nombreuses pendant l'internat (anesthésie-réanimation, chirurgie générale, gynécologie obstétrique). Dans ce deuxième groupe, figurent aussi «la médecine nucléaire, la neurologie, l'oncologie, la pédiatrie, la neurochirurgie, l'anatomie et cytologie pathologique, la pneumologie, l'endocrinologie [...], l'hématologie, la médecine physique et de réadaptation ainsi que trois spécialités plutôt exercées en mode libéral, la rhumatologie, la gynécologie médicale et la chirurgie orale» (Godefroy, 2013, p. 5).

À l'issue des ECN, la répartition des effectifs par sexe est fortement dissymétrique. En 2004, les femmes représentent près de $58 \%$ des étudiants affectés à la suite de ces épreuves mais seulement $29,5 \%$ de ceux optant pour les spécialités chirurgicales (BILLAUT, 2005). Aujourd'hui, dans certaines spécialités, «plus des trois quarts des internes sont des femmes : rhumatologie, pédiatrie, gynécologie-obstétrique, gynécologie médicale, endocrinologie, dermatologie. A contrario, elles sont moins de $40 \%$ parmi les internes de médecine nucléaire, neurochirurgie et chirurgie générale» (FAUVET, 2012, p. 8). Les spécialités chirurgicales sont ainsi, tendanciellement, parmi les moins choisies par les femmes. Alors que depuis 2007, les femmes représentent plus de $60 \%$ des internes (FAUVET, 2010), elles sont moins de $40 \%$ parmi les internes de médecine nucléaire, de neurochirurgie et de chirurgie générale.

Ainsi, la progression du nombre de femmes en chirurgie reste faible et peu en rapport avec la forte augmentation des effectifs féminins parmi les internes. La chirurgie dans son ensemble est donc peu attractive pour les femmes, notamment les chirurgies dites de base, très présentes dans les cursus d'apprentissage (chirurgie générale, viscérale et digestive, orthopédique). Les futures chirurgiennes arrivent dans un monde où elles sont peu nombreuses et où la mixité au sein des différentes spécialités n'est pas une réalité.

Derrière le fait que la chirurgie attire peu les femmes, se trouve un double enjeu social, à la fois d'inégalité et de non-mixité d'un métier de haut niveau. Si les femmes persistaient à ne pas choisir la chirurgie alors qu'elles obtiennent les meilleures places au concours, cela pourrait avoir pour conséquence de progressivement dévaloriser la discipline. En effet, comme nous l'avons vu, les femmes réussissent particulièrement bien aux ECN. Si elles persistent à peu opter pour la chirurgie, alors qu'elles sont parmi les premières dans 
les classements, cela pourrait conduire à ce que la chirurgie soit moins choisie par les meilleur-e-s classé-e-s au concours ${ }^{(3)}$. Cela induirait de manière quasi mécanique une perte relative du prestige de la chirurgie (Bercot, Mathieu-Fritz, 2007) alors qu'actuellement, les étudiants choisissent la chirurgie juste après les spécialités médicales. Tout en se caractérisant par un haut niveau de formation et un fort prestige social, le milieu des chirurgiens pratique par ailleurs un sexisme non euphémisé ( $c f$. infra) et constitue de ce fait une sorte d'enclave rétrograde qui peut être mise en regard avec les formes populaires du virilisme aujourd'hui les plus connues et les plus dénoncées (policiers, ouvriers, cf. infra).

Comment expliquer que la chirurgie soit si peu demandée par les femmes ? Plusieurs hypothèses ont été avancées pour tenter d'expliquer pourquoi les femmes ont tendance à ne pas choisir certaines spécialités médicales. Certains auteurs ont souligné que les femmes font les frais d'une stratégie d'exclusion de la part des hommes (CROMPTON et al., 1999); d'autres que les femmes préfèrent s'abstenir afin de pouvoir mieux gérer l'interface famille-travail (Crompton, Le Feuvre, 1997). Notre perspective est à la fois complémentaire et différente : nous mettrons l'accent sur l'importance de la socialisation dans le métier et ses effets. Différents travaux ont ainsi montré que c'est pendant leurs études que les médecins acquièrent les codes et connaissances propres à leur futur environnement professionnel et qu'ils apprennent à reproduire des schémas comportementaux hérités du passé. C'est également durant cette période que se construisent des identités sociales en lien avec la structuration interne du milieu. Marie JAISSON (2002), se référant aux travaux de Charles L. Bosk (1979), rappelle ainsi que dans la relation maître-élève s'exerce aussi un contrôle professionnel par l'inculcation d'une identité morale. La formation est essentielle tant du point de vue technique pour construire savoirs et compétences que pour inculquer les valeurs et les références propres au milieu. Or, dans la formation à la chirurgie, la relation maîtreélève et l'impact du maître sur l'élève sont d'autant plus forts que l'apprentissage se fait par compagnonnage. Non seulement l'acquisition des savoirs en dépend mais aussi la sélection à toutes les étapes de la formation à cette spécialité. Les études constituent donc un moment essentiel dans le processus de reproduction du milieu médical.

Il paraît utile en ce sens de revenir sur les modalités concrètes de la socialisation des jeunes à la

(3) Aujourd'hui, dans beaucoup de facultés, les internes doivent définir précisément leur future spécialité chirurgicale au tout début de leur internat et choisissent donc en même temps que la chirurgie, une spécialité précise, alors qu'antérieurement le choix de la spécialité pouvait se faire dans un deuxième temps. «Contrairement à ce qui se passait antérieurement, on ne peut plus cumuler plusieurs spécialisations » (JAISSON, 2002, p. 31). chirurgie afin d'éclairer sous un jour nouveau le sens que l'on peut attribuer à la quasi-absence des femmes de certaines spécialités chirurgicales (voir l'encadré pour la présentation de nos terrains d'enquête).

\section{Encadré \\ Les terrains investigués}

Notre analyse est tirée de plusieurs vagues d'enquêtes réalisées entre 2005 et 2010 . Une première enquête, financée par l'Assistance publique-Hôpitaux de Paris (AP-HP) et conduite en 2005-2006, est consacrée à la crise de recrutement des chirurgiens (entretiens réalisés avec Alexandre Matthieu-Fritz). Une deuxième enquête, financée par l'Observatoire national de la démographie des professions de santé (ONDPS), s'intéresse à la diversité des pratiques et des ressources chirurgicales. En 2009-2010, des entretiens ont été menés auprès d'autres catégories de chirurgiens que celles précédemment investiguées (chirurgiens du privé et spécialistes de la chirurgie infantile) et auprès d'internes en chirurgie : il s'agissait de comprendre comment et pourquoi ils avaient choisi telle spécialisation de chirurgie plutôt que telle autre (une partie de ces entretiens a été menée par Chantal Horellou-Lafarge). Dans cette dernière vague d'entretiens, nous souhaitions mettre à jour les difficultés rencontrées par les femmes, raison pour laquelle nous avons essentiellement interrogé des femmes.

Au total, 40 entretiens approfondis d' 1 h 30 à 2 h (de type semi-directif et biographique) avec des chirurgiens et des chirurgiennes ont été réalisés. Comme le résume le tableau 4, 18 l'ont été avec des chirurgiens en poste, 18 avec des internes et 4 avec des externes.

Les chirurgiens en poste ont retracé leur itinéraire et décrit leur rapport au travail et au métier.

Tableau 4 : Catégories de professionnels rencontrés en entretien

\begin{tabular}{|l|c|c|c|}
\hline $\begin{array}{c}\text { Catégories de professionnels } \\
\text { enquêtés }\end{array}$ & Hommes & Femmes & Total \\
\hline $\begin{array}{l}\text { Chirurgiens en poste dont* : } \\
\text { Neurochirurgie }\end{array}$ & 7 & $11^{* *}$ & 18 \\
$\quad$ Ophtalmologie & 1 & 3 & 3 \\
Chirurgie viscérale & 3 & 0 & 3 \\
$\quad$ Chirurgie orthopédique & 1 & 0 & 1 \\
Chirurgie esthétique & 0 & 2 & 2 \\
Chirurgie & 1 & 1 & 2 \\
$\quad$ otorhinolaryngologique & 0 & 1 & 1 \\
$\quad$ Gynécologie obstétrique & 1 & 3 & 4 \\
$\quad$ Chirurgie infantile & 3 & 15 & 18 \\
\hline Internes*** & 1 & 3 & 4 \\
\hline Externes & & \\
\hline
\end{tabular}

* Les statuts des chirurgiens sont divers : 16 sont salariés à l'hôpital. Parmi eux, on trouve 6 PU-PH (professeurs des universités-praticiens hospitaliers) et $10 \mathrm{PH}$, dont certains nous ont déclaré exercer une activité en libéral. Les 2 chirurgiens restant sont des libéraux. ** Six des femmes interrogées ont entre 40 et 45 ans; les cinq autres entre 55 et 63 ans.

*** Les internes rencontrés ont entre 25 et 31 ans.

Dans une première partie nous mettrons en évidence le fait que les femmes chirurgiennes incarnent une trajectoire d'émancipation sociale advenue très progressivement depuis 1984. 
La deuxième partie sera consacrée au cadre dans lequel a lieu l'apprentissage, principalement celui de la chirurgie viscérale ou orthopédique. Il en ressort qu'il peut être défini comme un monde communautaire par opposition au monde sociétaire qui est le cadre usuel de socialisation des jeunes en France. Nous étudierons tour à tour l'ethos et les dispositions qui se développent dans cette spécialité de manière spécifique par rapport à d'autres : la chirurgie viscérale notamment apparaît comme un monde organisé autour de la virilité et du sexisme, hostile aux femmes, et qui agit comme un répulsif.

Bien qu'assurant une grande part de la formation des étudiants, cette spécialité n'est pas révélatrice de la culture d'ensemble de la chirurgie. Il est essentiel d'envisager le monde de la chirurgie comme recouvrant une pluralité de mondes sociaux. C'est à cette condition que l'on peut montrer comment se construit la place des femmes: à quel prix, mais aussi selon quels chemins. Le regroupement des femmes dans certaines spécialités s'est de fait accompagné de la construction d'univers différenciés, plus ou moins attractifs pour les femmes. Nous soutiendrons la thèse selon laquelle il existe un autre ethos de spécialité chirurgicale, qui n'a jamais été décrit, ni fait l'objet d'études. Cet ethos jette les bases d'un modèle alternatif de socialisation et d'exercice du métier.

Dans une troisième partie, nous montrerons que les formes d'apprentissage en chirurgie viscérale sur le mode du compagnonnage supposent d'être adoubé. En nous basant sur le résultat de nos enquêtes, nous défendrons l'idée selon laquelle les modalités de l'enseignement par apprentissage nuisent gravement à la mixité en générant un mal être chez les femmes. Enfin, nous rendrons compte de la manière dont elles tentent d'accommoder ${ }^{(4)}$ et parviennent à aller au bout de leur formation en se «bricolant» une identité sociale provisoire.

\section{Se projeter dans des emplois et des études exigeantes : une trajectoire symbole de l'émancipation des femmes}

La socialisation peut être définie «comme le processus par lequel la personne humaine apprend et intériorise tout au cours de sa vie les éléments

(4) L'accommodation et l'assimilation sont deux mouvements complémentaires définis par Jean Piaget (1965) pour expliquer les manières dont se fait l'adaptation des individus à la société. L'accommodation consiste pour le sujet à se modifier pour répondre aux pressions et aux contraintes de son environnement. Par l'assimilation, le sujet chercherait à modifier son environnement pour le rendre plus conforme à ses désirs. Les individus s'ajustent ainsi continuellement aux changements dans l'environnement. socioculturels de son milieu, les intègre à la structure de sa personnalité sous l'influence d'expériences et d'agents sociaux significatifs et par là s'adapte à l'environnement social où elle doit vivre» (ROCHER, 1968, p. 162). L'analyse de ce processus doit prendre en considération des facteurs à la fois macro- et microsociaux. Aussi, pour étudier l'entrée dans le métier de chirurgien, il nous faut revenir sur quelques éléments de cadrage sociétaux concernant la place des femmes, qui font partie intégrante des conditions et modalités de la socialisation que nous avons observées. Aujourd'hui, dans le contexte de la modernité, les modes de socialisation sont divers et ouverts dans la mesure où une pluralité d'acteurs et de médias y participe. Reconsidérer le chemin parcouru par les femmes nous permettra de mettre en exergue la spécificité du monde de la chirurgie viscérale dont le caractère communautaire et misogyne constitue un cadre de domination et de dévalorisation des femmes qui joue un rôle central dans les apprentissages. Nous faisons l'hypothèse qu'un tel contexte a des effets sur les étudiants même s'il est difficile de mesurer à quel point il joue sur leur choix d'opter ou non pour la chirurgie.

\section{Des étudiantes puis des femmes brillantes}

Avoir la possibilité de s'orienter vers la chirurgie à l'issue des ECN signifie que les candidats ont suivi un parcours d'études supérieures d'excellence : ils ont réussi le concours d'entrée en médecine puis ont été classés parmi les premiers aux ECN. Les chirurgiennes que nous avons rencontrées ont pour beaucoup été reçues lors de leur première tentative au concours d'entrée, alors que certaines étaient encore très jeunes. Sûres de leurs capacités intellectuelles qu'elles ont pu comparer à celles des garçons qu'elles ont côtoyés dans leur cursus dans le secondaire, elles ont obtenu un bon classement aux ECN, qui leur a permis d'opter pour la chirurgie, alors la deuxième spécialité la plus choisie après les spécialités médicales ${ }^{(5)}$.

La voie leur a été tracée par des chirurgiennes plus âgées qui, elles, ont été élevées dans une société où les femmes étaient encore sous l'autorité masculine. Pour autant, ces pionnières de la chirurgie n'ont reçu ni de la part de leur mère (qui étaient en général des femmes actives) ni de celle de leur père l'éducation classique de leur époque. D'après les propos de certaines que nous avons interrogées, elles ont été élevées comme des garçons, ou sans que leurs parents semblent accorder d'importance au genre; 1'objectif poursuivi était leur épanouissement personnel et non qu'elles deviennent épouse ou mère : "J'ai été élevée comme un garçon, [...] et j'ai découvert très tardivement qu'on n'avait rien à voir, qu'un homme et une femme, c'est

(5) Ceci est resté vrai jusqu'en 2004 (Billaut, 2005). Les résultats indiqués plus haut pour l'année 2012 montrent toutefois qu'ils varient selon les types de chirurgie. 
complètement différent» (Chirurgie esthétique, libéral, PU, femme, 61 ans). L'important était d'être indépendante :

«Mes parents ont toujours dit: fais le métier qui te plaît d'une part et aie un métier pour avoir ton indépendance financière, ne jamais dépendre de quelqu'un; fais ce qui te plaît, et fais-le bien. [...] Je suis le pur produit de ma mère et de mon père qui a donc beaucoup, je pense, investi intellectuellement sur moi ; donc, entre un père polytechnicien et une ingénieure chimiste. »

(Chirurgie réparatrice, libéral, PU, femme, 63 ans.)

Prendre conscience qu'il est possible de rester femme tout en jouant le rôle et en ayant les compétences d'un chirurgien a demandé à ces femmes un travail sur elles-mêmes. À leur époque, elles ont dû renoncer à «être une femme» ou courir le risque ne pas être prises au sérieux :

«La voie est étroite pour une femme: ou vous ressemblez à une minette, et vous êtes moyenne, "bof chirurgien" mais c'est une femme! Ou vous faites votre métier de façon impeccable et vous ressemblez à une armoire à glace. On dit : "Ah ben d'accord, c'est un bon chirurgien mais c'est pas une femme"."

(Chirurgie réparatrice, libéral, PU, femme, 63 ans.)

Elles opposent ainsi le fait qu'on puisse satisfaire tout à la fois aux différents critères professionnels et à ceux du genre tels qu'ils sont pensés dans leur entourage.

Les rares chirurgiennes de cette génération arrivées au sommet de la hiérarchie ont dû adopter un comportement particulier pour se faire admettre dans leur milieu professionnel; les plus jeunes les décrivent d'ailleurs parfois comme caractérielles, hystériques, ayant tout donné à la chirurgie. Elles font figure de contre-modèles pour les femmes des générations suivantes qui souhaitent s'épanouir dans cette profession sans renier leur appartenance de genre.

\section{Redéfinition des rôles familiaux par les jeunes chirurgiennes}

Les plus jeunes, pour lesquelles il semble normal pour une femme de travailler, revendiquent le droit de se passionner pour un métier aussi prenant soitil. Non seulement leur activité ne se réduit plus à l'apport d'un salaire d'appoint mais elles ne se définissent plus comme le pivot central de la famille. L'évolution des mentalités leur permet de défendre une conception plus individualiste de leur vie et d'adopter un modèle d'interaction conjugale qualifié d'hypoconjugalité (CommaILle, 1993): leurs compagnons doivent désormais composer avec leurs stratégies professionnelles (LAPEYRE, Lefeuvre, 2005). En cela, comme leurs aînées, elles ont été soutenues voire encouragées par leur père et leur mère. Ainsi qu'en témoignent les extraits d'entretiens ci-dessous, le soutien des parents est capital pour l'insertion future de ces jeunes femmes dans le milieu professionnel ; il l'est d'autant plus qu'il s'agit d'un milieu masculin.

"Quand j'étais petite, un jour, en jouant à l'arrière de la voiture, je disais que je voudrais être infirmière et mon père m'a dit: "Pourquoi infirmière? Tu seras médecin."”

(Chirurgie vasculaire, interne, femme, 26 ans.)

Une urologue se souvient de son père regardant un défilé du 14 juillet :

"Quand on voyait les filles de Polytechnique ou de Saint-Cyr, il me disait: "Regarde, c'est extraordinaire; regarde, maintenant il y a des filles dans le défilé." "

(Urologie, interne, femme, 28 ans.)

Tout en exerçant le métier qu'elles aiment, ces femmes renoncent souvent à même convoiter les postes les plus élevés, ce qui leur permet de ne pas être en concurrence avec leurs confrères. Cette posture de compromis ne va pas sans tensions pour celles qui ont de l'ambition et souhaitent faire une belle carrière ou prendre de plus hautes responsabilités: tension au travail mais aussi tension hors travail. En effet, si l'évolution de la société a conduit certains hommes, dont la plupart ont vu leur mère travailler, à participer aux responsabilités familiales et à accepter, voire à encourager leur compagne dans son travail, aussi lourd et chronophage soit-il, il n'en demeure pas moins que ce sont encore souvent les femmes qui assument la charge $\mathrm{du}$ fonctionnement quotidien du ménage. L'enjeu est donc pour elles de parvenir à trouver un équilibre mouvant entre vie de travail et responsabilités familiales. Certaines internes se félicitent ainsi que leur compagnon ne soit pas médecin, ce qui leur permet d'assurer les astreintes et les gardes sans problème; d'autres, qui sont femmes de médecins, considèrent qu'elles trouveront toujours un moyen de faire garder leurs enfants soit en payant du personnel à domicile, soit en faisant appel à leur famille. Elles envisagent leur future vie familiale comme le résultat d'une négociation avec leur conjoint qui leur permettra de continuer à pratiquer leur profession comme elles l'entendent ${ }^{(6)}$.

(6) Les femmes en poste ont évoqué également leur vie de famille. Les femmes des générations les plus anciennes disent avoir peu vu leurs enfants et les avoir confiés à des nourrices. Les femmes des générations suivantes s'appuient également sur du personnel rémunéré venant à domicile. Néanmoins, on constate des arrangements différents en fonction des jours de la semaine. Ainsi, lorsqu'elles n'ont pas d'intervention, elles assument la responsabilité des incidents potentiels (maladie d'enfant, questions diverses d'intendance au domicile). Concrètement, cela signifie qu'elles se déplacent en personne pour régler le problème ou bien qu'elles trouvent quelqu'un pour le faire à leur place. Certains autres jours de la semaine et notamment lorsqu'elles ont une intervention, ce sont leurs conjoints hommes qui prennent le relais. Les hommes, quant à eux, évoquent peu la question des arrangements familiaux, si ce n'est pour dire que les responsabilités domestiques sont à la charge de leurs épouses. 
Ces femmes, qui ont déjà fait la preuve de leur ténacité à l'issue d'une trajectoire de formation témoignant de capacités intellectuelles et de travail hors normes, se projettent volontairement vers des métiers exigeants et se retrouvent face à un monde qui apparaît anachronique par bien des traits.

On peut analyser de nombreuses traditions observées lors de l'internat en médecine comme construisant un rapport au corps - qu'il s'agisse de celui de l'autre ou du sien propre. L'ensemble du monde médical est l'héritier d'une histoire qui tente de dédramatiser les problèmes de santé. Le corps, de ce fait, est souvent réifié et le malade mis à distance pour mieux neutraliser les émotions. $\mathrm{Au}$ cours de la formation, l'«objectivation du corps », ainsi nommée par Emmanuelle Godeau (2007, p. 190), se fait par des jeux qui portent sur les parties du corps les plus problématiques et embarrassantes à examiner pour les jeunes apprentis : les organes sexuels et l'appareil génito-urinaire. Des baptêmes ou des cérémonies consistent en des mises à l'épreuve des corps, et des organes génitaux en particulier ${ }^{(7)}$. Selon Pierre Bourdieu (1982, p. 59), «l'efficacité symbolique des rites d'institution [réside dans] le pouvoir qui leur appartient d'agir sur le réel en agissant sur la représentation du réel». On peut donc faire l'hypothèse que ces rites sont non seulement destinés à sceller l'appartenance des étudiants au monde médical mais qu'ils ont aussi pour fonction de les aider à supporter la violence que représente l'intrusion dans les corps.

L'humour de carabin contribuerait par ailleurs à un maintien des référents virils en lien avec la dimension héroïque du métier. Par le passé, la création de mimes et de chansons paillardes faisait aussi partie du bizutage. Des épreuves à caractère obscène conduisaient les étudiants à pratiquer l'exhibitionnisme ou la suggestion sexuelle. Ces épreuves n'ont aujourd'hui pratiquement plus cours, de même que les invitations de prostituées dans les salles de garde. L'arrivée massive des femmes a en effet modifié les pratiques. Cependant, évoquant les rites et les cérémonies de l'internat, GoDEAU souligne que, malgré la féminisation des promotions, les garçons continuent de donner le ton des rites et de l'ambiance des salles de garde.

(7) Godeau (2007, p. 191) écrit par exemple : «[...] l'ingestion (à l'insu des victimes bien évidemment) de diurétiques ou de laxatifs, entraînant une régression forcée à un stade où l'on ne maîtrise plus ses fonctions physiologiques élémentaires, réduisant le vécu corporel aux dérèglements d'un corps mécanique. En confrontant, "pour rire" leurs camarades à cette dépendance à l'égard du corps dans ce qu'elle a de plus humiliant et d'angoissant, les aînés ne leur indiquent-ils pas aussi la stratégie privilégiée de leur futur corps professionnel face à de telles expériences : l'humour et la dérision?»

\section{La chirurgie viscérale définie comme monde communautaire et les autres chirurgies : quelles dispositions ?}

Le monde des chirurgiens viscéraux nous est apparu comme un monde à part en raison de ses modes de fonctionnement mais aussi de la manière dont il se situe par rapport aux autres spécialités. Après avoir décrit le monde communautaire de la chirurgie viscérale et son ethos, nous rendrons compte des effets qu'il exerce sur les dispositions en mettant l'accent sur différentes spécialités chirurgicales.

\section{Monde communautaire et ethos}

La chirurgie viscérale est un monde communautaire tel que l'entend Ferdinand TönNIEs (1922), pour qui la communauté englobe tous les modes de relations sociales et toutes les formes d'organisation sociale caractérisées à la fois par des liens affectifs étroits, profonds et durables, par un engagement de nature morale et par une adhésion commune à un groupe. La communauté peut être celle du sang ou de l'esprit. La communauté «chirurgie viscérale» a des valeurs et des normes spécifiques car elle a, d'une part, une conception qui lui est propre de l'appartenance sociale au groupe et du rapport à l'autorité, et car, d'autre part, le statut et la place accordés aux femmes y sont bien particuliers. Si l'inscription sociétale signifie diversité des valeurs, des attachements et des investissements (Simmel, 1999), l'inscription communautaire s'appuie a contrario sur des références, des langages, des valeurs et des comportements partagés, qui se construisent, pour la communauté des chirurgiens comme pour les autres, par des apprentissages à la fois concrets et sociaux.

Segment le plus ancien de la chirurgie, qui se confondait autrefois avec la chirurgie générale, les chirurgies viscérale et orthopédique sont nées et se sont développées sur les champs de bataille durant les guerres. Même si les chirurgiens actuellement en activité n'ont pas connu cette époque, la culture militaire continue à imprégner la profession. L'emprise hiérarchique est ainsi très présente, et quotidiennement visible: les tâches les moins valorisantes, le dirty work (Hughes, 1996), sont ainsi confiées aux plus jeunes comme s'il s'agissait là de signes d'allégeance : il leur est par exemple demandé de transporter des dossiers ou de rendre de menus services n'ayant pas grand-chose à voir avec leur apprentissage d'interne; ils sont censés tout accepter ainsi qu'en témoigne l'extrait d'entretien suivant :

"L'interne, il doit tout accepter ou alors, c'est qu'il $n$ 'a rien compris. Le compagnonnage, c'est particulier, on peut dire ce qu'on veut... ça s'arrangera 
toujours [sous-entendu, les tensions s'arrangeront toujours] ... On est de la même famille [...]. Il [1'interne] a choisi de faire chirurgie, c'est un contrat, on lui apporte tout, on est ses supérieurs; s'il n'est pas content, c'est pareil.»

(Chirurgie viscérale, $\mathrm{PH}$, homme, 44 ans, hôpital public.)

Cet extrait reflète à quel point un état d'esprit bien particulier est de mise en chirurgie viscérale, au sein de laquelle règne l'autorité inconditionnelle du maittre. Les relations entretenues entre les internes et leurs maîtres sont comparées à des relations familiales dont la conception, singulière, a peu à voir avec les normes de notre société actuelle.

La chirurgie, métier qui cohabite avec la mort, a, notamment pour ceux qui la pratiquent, une dimension héroïque non négligeable. En outre, l'ethos qui s'y observe se caractérise par des représentations dichotomiques des rôles et des qualités à assigner aux femmes et aux hommes dans la société. L'attitude dominatrice et sexiste des hommes y est rendue possible par la conjonction de trois phénomènes : il s'agit d'un monde relativement fermé; les femmes y sont minoritaires; les hommes y occupent une place de pouvoir exclusive. On retrouve ici les caractéristiques repérées dans d'autres mondes masculins tels que la police (PRUvost, 2007), ou plus largement chez les ouvriers (CROMER, LEMAIRE, 2007).

Dans cet ethos, les femmes sont perçues comme devant rester attachées à la sphère familiale («le dedans» opposé à «l'extérieur», pour paraphraser Françoise HÉRITIER [1996]). Des chirurgiens du viscéral nous affirment lors des entretiens que les femmes n'ont pas leur place dans ce métier: elles sont censées permettre aux hommes d'exercer leur travail dans les meilleures conditions en leur épargnant tout souci domestique et toute préoccupation liée à l'éducation des enfants. Parmi les chirurgiens rencontrés, certains disent explicitement ne s'être jamais occupés du quotidien de la vie de famille, ne jamais y avoir pris la moindre part; ils justifient cette absence par leur investissement dans leur activité de travail et la lourdeur des responsabilités qui leur incombent; ils ne peuvent imaginer qu'une femme puisse faire face à une telle charge de travail et se décharger sur son compagnon des tâches domestiques.

\section{L'attrait d'une fonction : les étapes d'un choix}

Pour quelles raisons alors les femmes choisissentelles la chirurgie ? Notamment parce qu'elles ont croisé la trajectoire de femmes ayant réussi dans la profession, ce qui les a confortées dans leurs aspirations :

"C'est une femme qui m'a donné envie de faire de la chirurgie. Ça a été une rencontre très importante. [...] [Il s'agit d'] une femme exceptionnelle, que je rencontre toujours. [...] Elle avait un dynamisme terrible. Elle n'était pas facile, elle avait un caractère... Elle n'était pas souriante au premier abord, mais elle avait un dynamisme, une passion pour ce qu'elle faisait et elle était extrêmement brillante. »

(Chirurgie viscérale, $\mathrm{PH}$, femme, 50 ans.)

En cela, elles ne sont guère différentes de leurs confrères qui se réfèrent souvent à des figures leur ayant servi de modèle.

Nos enquêtés sont parvenus à la chirurgie viscérale par deux voies principales. Certains ont décidé de s'orienter vers la chirurgie au début de leur internat sans savoir encore vers quelle spécialité chirurgicale ils se tourneront dans la suite de leur parcours. En général, ces étudiants ont vécu des expériences fortes et diversifiées en chirurgie avant même leur internat, si bien qu'ils savent vouloir devenir chirurgiens. Ils souhaitent «mettre les mains dedans », ouvrir, pénétrer dans le corps et accomplir les gestes techniques. D'autres étudiants ont au départ un intérêt particulier pour un organe ou une population (l'œil, les enfants, etc.). Ils décident de faire chirurgie dans un deuxième temps, à la suite d'un stage au cours duquel ils découvrent le travail au bloc. Quelles que soient les étapes qui conduisent les étudiants à choisir la chirurgie, l'expérience acquise en stage est essentielle. Pourtant, au stade de l'externat, le stage de chirurgie est peu prisé, car il prépare mal au concours des ECN et présente un faible intérêt pour les externes qui y «voient» peu de choses et ne peuvent intervenir. Il permet néanmoins de découvrir l'atmosphère des blocs et les relations qui se nouent autour de l'intervention. Lorsqu'ils n'ont pas effectué de stage, les étudiants s'appuient sur la réputation de certains services de chirurgie pour faire leur choix. Or, leur représentation du monde de la chirurgie se construit principalement à partir de ce qu'ils savent et apprennent des chirurgiens viscéraux et orthopédiques, qui occupent une place prédominante dans les parcours d'apprentissage des jeunes.

\section{Des dispositions contrastées selon les mondes sociaux}

L'ethos qui vient d'être décrit interfère avec les modalités de mise en œuvre d'un certain nombre de dispositions mobilisées pour la chirurgie. Joan Cassell (2000) souligne les dimensions culturelles sur lesquelles s'appuient les représentations de métiers très masculinisés comme celui de la chirurgie: dynamisme, force, esprit de décision, courage, agressivité. On peut aussi y ajouter l'endurance physique et morale. Nous discutons ci-après les dispositions qu'Emmanuelle Zolesio a mises en évidence dans sa recherche sur la socialisation en chirurgie viscérale (ZoLesIo, 2012) : dispositions à l'action, à la confiance en soi et au leadership, à l'endurance physique, mais aussi absence d'appétence pour la relation au patient. Nous les comparerons 
avec nos propres résultats et notamment avec les modalités présentes dans d'autres spécialités, ce qui nous permettra de mettre en évidence l'existence de dispositions alternatives valorisées dans d'autres spécialités chirurgicale.

La disposition à l'action se traduit par une volonté de rapidité, un grand dynamisme, une dépense d'énergie conséquente, et par un «côté hyperactif» (ZoLEsio, 2012, p. 380). L'importance accordée au geste peut également y être rattachée. C'est en effet souvent ce qui a fasciné les internes au moment de leur orientation. Contrairement à d'autres spécialités, la chirurgie offre une solution immédiate et visible aux problèmes posés. Cette capacité à intervenir pour transformer non dans la durée mais dans l'instant l'état de santé du patient est fortement mise en avant dans le choix de la discipline. L'acte est un stimulant; il est source d'intérêt car il s'accompagne d'un challenge personnel fort. Il repose sur la réactivité et l'immédiateté de la prise de décision (KATZ, 1999; BerCOT, MATHIEU-Fritz, 2007 ; ZOLESIO, 2012).

Cependant, dans les services de chirurgie infantile, si les femmes insistent sur le geste, les modalités d'action et de décision, elles ne désignent ni l'agressivité ni la force comme des traits culturels nécessaires mais mettent en exergue les qualités inverses : la patience et l'endurance.

En outre, dans toutes les spécialités chirurgicales, qu'il s'agisse de l'orthopédie, de la chirurgie viscérale, de l'ophtalmologie ou de la chirurgie infantile, les professionnels soulignent qu'il leur apparaît nécessaire de savoir prendre des risques, qu'ils s'appliquent à mesurer au mieux et qui s'accompagnent toujours d'une incertitude, génératrice de stress, quant à l'issue de l'intervention, comme l'indique le témoignage suivant :

"Rien n'est grave dans l'activité, sauf les malades, lorsqu'il y a risque vital. Il y a très peu de différence entre une opération qui se passe bien et une opération qui ne se passe pas bien... Mais il y a des choix, des arbitrages à faire quand tout ne se passe pas comme on voudrait. Par exemple une personne qui a de l'embonpoint : il faut décider quelle prothèse on met, comment on l'adapte... le choix doit être fait dans l'immédiat, on est dans le faire, c'est un métier de décision. En opération, on doit faire dans l'urgence, on ne peut prolonger l'opération, on a derrière un programme de consultations, cela oblige à faire rapidement [....]. Une difficulté est de gérer le stress. Certains explosent dans les blocs opératoires, c'est un moyen de renvoyer le stress vers les autres. Il y a beaucoup d'engueulades dans les blocs opératoires. Le chirurgien est en instabilité quand il est en bloc opératoire. Il a des conduites de réassurance car il doit choisir un instrument et cela le met dans un état instable. Il a des gens entre les mains. [...] Il n'y a pas de routinisation, pas d'assurance. On peut merder sur chaque opération. Il est nécessaire d'apprécier la dureté de l'os, la manière dont la fraise est aiguisée, il faut choisir la place de l'incision... Il est intéressant de rechercher une industrialisation du process pour mieux le maittriser mais le process garde un caractère d'artisan avec des aléas. 》 (Chirurgie orthopédique, PU-PH, homme, 50 ans.)

Les femmes indiquent qu'il est important pour elles de s'autoévaluer de manière correcte. Ce faisant, elles parviennent à gagner en confiance. Une jeune interne explique ainsi :

"Il faut savoir ses limites tout en ne se dévalorisant pas et j'aurais tendance à dire que les femmes, on est moins douées là-dessus; on a toujours tendance à se dévaloriser; moi, je suis toujours contente quand ça marche bien, mes collègues hommes ont toujours tendance à se la ramener; moi, ce n'est pas du tout ça. »

(Chirurgie maxillo-faciale, interne, femme, 28 ans, 3e année.)

Une telle attitude joue aussi dans la construction de la carrière dans la mesure où les femmes se mettent moins en avant que les hommes.

La confiance en soi et le leadership constituent une autre disposition partagée par les professeurs en chirurgie viscérale ou en orthopédie. Certains internes en font aussi preuve, qui mettent en avant le caractère héroïque du métier dont ils tirent un certain orgueil (Zolesio, 2012, p. 381). Cette manière d'être, autoritaire et sûr de soi, ferme dans ses volontés, défendant avec force son point de vue et ses intérêts, se retrouve dans différentes disciplines de chirurgie. Elle est associée à l'image du patron, mais ne s'exprime pas toujours selon les mêmes modalités. En effet, dans les négociations avec les cadres infirmiers et les infirmières, les hommes chirurgiens peuvent adopter des postures très diverses. Certains campent sur leur point de vue sans faire aucune concession, considérant que l'espace du bloc opératoire leur appartient en propre, et refusent de se plier à toute régulation autre que celles qu'ils ont instituées. D'autres, au contraire, tentent de concilier les intérêts de toutes les parties, y compris ceux des personnels paramédicaux qui gèrent le bloc opératoire. Les cadres de santé et les infirmières, tout en soulignant à quel point certaines personnalités sont odieuses, signalent toutefois des appuis et des collaborations possibles (BERCOT, 2009). En outre, lors de certains entretiens, nos interlocuteurs ont fait preuve d'extrême modestie vis-à-vis de leurs propres compétences et d'une grande déférence envers des pairs qu'ils considèrent comme des maîtres; tel fut par exemple le cas d'une femme PU-PH, chef de service en neurochirurgie, elle-même très estimée et faisant référence dans son domaine. Plusieurs internes en ophtalmologie, en chirurgie infantile ou en neurochirurgie se sont montrés extrêmement humbles : lucides sur la difficulté à maîtriser les savoirs et à tirer tous les enseignements de leurs différentes expériences, ils sont conscients du chemin qu'il reste toujours à 
parcourir dans l'acquisition des connaissances, y compris pour les meilleurs.

Complémentaire de la confiance en soi, une autre dimension, très présente dans plusieurs chirurgies et notamment en chirurgie viscérale, est régulièrement mentionnée par les chirurgiens. Il s'agit de la capacité à affronter la mort toujours possible d'un patient, ou l'infirmité qui peut découler d'une intervention non réussie. Voici ce que dit un chirurgien viscéral de la manière dont il appréhende la mort :

"Quand c'est votre malade, il y a de l'affectif; le pluridisciplinaire aide à prendre une décision... La mort sur la table est souvent attendue. [...] On n'est pas à l'abri aussi d'un arrêt cardiaque non attendu. On a la tête prise [également] chez soi. Quand un malade ne va pas bien, j'y pense tout le week-end. Mettre à distance le stress, ça s'apprend. Au début, on a peur de toutes les interventions, même les banales, on a peur d'un incident. On apprend à le gérer. On prend de l'assurance mais il y a encore du stress, mais on prend de l'assurance. J'ai l'impression d'être au sommet : j'ai acquis une plénitude dans ce que je sais faire, je connais mes limites. Je les connais mieux qu'il $y$ a dix ans. C'est fatigant physiquement et psychiquement. Il n'y a pas d'ergonomie au travail: j'ai eu une sciatique pendant un an; on prend pas d'arrêt de travail, on se donne [nous soulignons pour mettre en valeur le ton appuyé]. »

(Chirurgie viscérale, PH, hôpital public, homme, 44 ans.)

La disposition «endurance physique» est indispensable pour des spécialités telles que la chirurgie viscérale, la chirurgie infantile ou la neurochirurgie, où les interventions peuvent être très longues (plus de douze heures parfois en neurochirurgie). Elle apparaît moins fondamentale dans d'autres spécialités comme l'ophtalmologie, qui valorisent plutôt l'extrême précision et la forte spécialisation sur certaines opérations.

Une autre dimension concerne la disponibilité temporelle : les chirurgiens doivent pouvoir assurer une présence continue. Or, cette dimension nous est apparue de manière un peu différente de celle décrite dans d'autres travaux (Zolesio, 2012) : indissociable de l'ethos viril, il peut s'agir d'un moyen d'exclure les femmes en montrant que le métier de la chirurgie est incompatible avec leur rôle social. Le témoignage ci-dessous illustre l'importance accordée à cette disponibilité temporelle :

«Les anesthésistes ont obtenu du repos compensateur après la garde; mais les chirurgiens, eux, restent car ils sont responsables des malades. Les anesthésistes n'ont pas la même responsabilité; ils disent: "C'est ton malade" au chirurgien lorsqu' il y a un problème et donc, c'est au chirurgien de dialoguer avec le malade et la famille, de lui annoncer les problèmes. Les anesthésistes sont "couverts" par les chirurgiens; cela ne pose aucun problème à ces derniers. Au contraire, ils n'envient pas cette posture de retrait, de nonresponsabilité. Les complications sont gérées par les chirurgiens, ils sont plus responsables. Mais cela a un avantage: c'est que les gens leur font confiance et ce sont les chirurgiens qui reçoivent des remerciements. »

(Chirurgie viscérale, PH, hôpital, homme, 44 ans.)

Comme nous l'avons déjà évoqué, l'enjeu de la disponibilité temporelle pose la question de la place des femmes. Des chirurgiens en chirurgie viscérale nous ont affirmé lors des entretiens que les femmes n'y ont pas leur place et avancent pour cela deux arguments. Le premier concerne la longueur des interventions (parfois jusqu'à douze heures), l'endurance qu'elles requièrent, mais également la force, en chirurgie orthopédique notamment: ce n'est donc pas un métier pour les femmes, l'endurance ne pouvant être féminine mais uniquement masculine... Rappelons à toutes fins utiles que les femmes sont nombreuses en chirurgie infantile; or, comme la chirurgie viscérale, il s'agit d'une chirurgie générale, certes appliquée à une population différente mais où les interventions sont également très longues. Une telle argumentation ignore en outre le développement de la célioscopie qui nécessite plus d'habileté que de force. Alors que l'habileté est une qualité par ailleurs souvent attribuée aux femmes et qu'elle est d'une importance primordiale en chirurgie, elle ne leur est ici pas reconnue ${ }^{(8)}$. Le second argument mis en avant pour écarter les femmes concerne les temps de travail qui seraient incompatibles avec le rôle qu'elles sont censées jouer dans notre société. Cet argument est essentiel. En réponse à nos questions, les chirurgiens viscéraux ont assuré qu'aucun aménagement du temps de travail n'est possible, de même qu'aucune délégation de responsabilité sous peine de mal servir le patient et de prendre des risques pour sa santé. En médecine, la référence au patient est souvent utilisée pour légitimer des pratiques professionnelles (Hughes, 1996). Les titulaires, parmi les plus hauts dans la hiérarchie, déclarent assumer l'ensemble du processus d'intervention, seuls, dans la durée. Or, dans les faits, la délégation tout comme la coopération avec des collègues ou des internes existe. Les internes recousent et peuvent assurer le suivi postopératoire tandis que les chefs de clinique( ${ }^{(9)}$ prennent le relais sur certaines interventions.

Des chirurgiennes s'attaquent néanmoins à la chirurgie en tant qu'archétype du métier «viril», repensent le métier, travaillent à faire accepter une grossesse et s'emploient à aménager une vie familiale :

«En fait, c'est une question d'organisation. Il y a un an, moi, j'ai beaucoup pleuré parce que je me suis dit : j'ai choisi un métier qui va m'empêcher d'avoir une vie de famille; j'étais persuadée il y a un an que

(8) À l'inverse, elle a souvent servi d'argument pour utiliser des femmes ouvrières sur certains postes industriels.

(9) Ce grade hospitalier correspond à celui qui est immédiatement en dessous de celui de chef de service. 
c'était impossible de faire les deux. [...] Maintenant que je vieillis un peu je me dis : 1) c'est une question d'organisation; 2) une question d'argent... Maintenant, je n'ai vraiment aucune inquiétude; autant j'ai des angoisses sur mes compétences de chirurgien de temps en temps, autant pas du tout sur le fait d'arriver à mener...»

(Urologie, PH, hôpital, femme, 36 ans ${ }^{(10)}$.)

C'est ainsi que des modalités différentes de rapport au temps, incarnant un ethos alternatif à celui habituellement mis en avant, concernent en réalité des services entiers. Ainsi, en chirurgie infantile, nous avons observé qu'un autre modèle de gestion du temps pouvait être mis en œuvre. Le travail, tout en faisant l'objet d'une forte mobilisation, n'y est pas sous-tendu par des normes rigides. Les chirurgiennes effectuent de longues journées de travail, tout comme leurs collègues masculins, mais on y constate dans le même temps un droit assumé à l'organisation conjointe des horaires afin de préserver la vie hors travail, tant pour les hommes que pour les femmes. Les temps de présence et l'organisation des journées sont ainsi réaménagés et ajustés en conséquence et pour tous les membres des équipes. Même si les temps de travail restent conséquents (les femmes peuvent y assurer douze heures de travail et assumer l'ensemble des gardes de leur spécialité), la durée du travail n'apparaît pas, dans ces services, comme une norme intangible; elle n'y est pas requise, ni revendiquée comme un trait distinctif voire honorifique du métier. Cette approche différente et plus tolérante du temps de travail ne remet nullement en cause la professionnalité de ceux qui en bénéficient et permet de répondre aux aspirations des femmes mais aussi des hommes de la génération actuelle qui souhaitent mener une vie en dehors du travail. Ce rapport nouveau à l'organisation du travail et au temps représente en soi une dimension d'un modèle alternatif de l'ethos au travail en chirurgie viscérale ou orthopédique.

Enfin, l'importance accordée aux dispositions relationnelles varie en fonction des spécialités. Nos interlocuteurs mettent en avant que le malade qui accepte de leur confier son corps et de se soumettre à l'intrusion que représente l'intervention fait preuve d'une grande confiance à leur égard - et ils s'en sentent valorisés. Une interne nous explique ainsi :

"J'ai découvert la chirurgie dans les stages : il y a un côté efficace, pratique, les résultats sont immédiats quand on opère et puis, il y a le côté technique. [...] Il y a une confiance, il [le patient] se confie à vous, il est endormi et livre son corps; c'est incroyable cette confiance. [...] Est-ce qu'ils se rendent bien compte de la confiance qu'ils nous font en acceptant l'opération?»

(Ophtalmologie, interne, 5e année, femme, 30 ans.)

(10) À titre de comparaison, voir ZoLEsIo (2011) sur la période de la grossesse en chirurgie digestive.
Dans ses écrits, Zolesio montre qu'en chirurgie viscérale, le patient ne fait l'objet que de peu d'attention et que le chirurgien lui consacre un temps très limité. Les récits que nous avons recueillis, de même que les descriptions critiques que les internes nous ont fait des visites, confirment qu'elles ont lieu au pas de course. Seule l'observation peut rendre compte de comportements portant atteinte à la pudeur du patient ou de modes de communication qui le bousculent. Il faudrait la compléter par des observations dans d'autres mondes chirurgicaux, notamment la chirurgie infantile. En effet, nos observations en ophtalmologie montrent que la relation au patient est très différente : ce dernier est conscient pendant l'intervention, il importe donc de le ménager. C'est pourquoi les échanges entre intervenants durant les opérations sont codés et les discussions préopératoires avec le patient habituelles. De même, le travail en chirurgie infantile implique la construction d'un dialogue triangulaire entre parents, chirurgiens et enfants, ce qui conduit les chirurgiennes que nous avons interrogées à prêter beaucoup d'attention à la relation avec les enfants.

Tout comme leurs homologues masculins, les femmes qui choisissent d'être chirurgiennes en dépit de l'accueil réticent qui leur est réservé, ont besoin d'être guidées dans leur apprentissage du métier par des chirurgiens experts. S'apparentant à une forme de compagnonnage, cet apprentissage met la question des interactions au cœur de l'enseignement.

\section{Être adoubée, condition pour apprendre le métier}

Intéressons-nous maintenant aux conditions dans lesquelles ont lieu les apprentissages. Le professionnel est amené à faire preuve de virtuosité aux différents moments de la chaîne d'intervention: lors du diagnostic, de la décision d'opérer, lors de l'intervention elle-même, et enfin, lors du suivi postopératoire. Les internes font leur apprentissage en se frottant à des situations concrètes et en effectuant eux-mêmes des interventions. Les conditions sociales de cet apprentissage - et notamment le sexisme qui règne dans certains services - ne tissent pas qu'une toile de fond mais interfèrent sur les modalités de l'interaction entre les apprenants et leurs maîtres.

\section{L'acquisition des savoirs suppose d'être adoubée}

Apprendre à opérer signifie acquérir des connaissances théoriques mais aussi apprendre à se servir de son corps en intervenant avec habileté, sans se tromper et sans endommager les différents organes du patient. La dextérité du chirurgien se construit au fur et à mesure de son apprentissage, par la pratique et au fil des interventions sur les corps : 
«L'enseignement se fait progressivement, le sensoriel passe par la pratique. Par exemple, écarter avec une certaine force mais sans nuire. L'étudiant doit faire un travail personnel. »

(Chirurgie orthopédique, PU-PH, homme, 50 ans.)

Les acquisitions ne peuvent donc se faire que progressivement, grâce à l'accompagnement constant des seniors. À l'issue d'une phase d'observation, succédera l'acquisition de gestes grâce à des conseils et à «la conduite de la main» par les plus expérimentés. Ils effectuent ensemble des interventions qui permettent aux jeunes d'acquérir de l'expérience : ils y apprennent à maîtriser aussi bien les risques encourus que leurs émotions, et à se concentrer sur des temps parfois très longs. Les internes sont amenés à se bâtir une solide confiance en eux-mêmes, nécessaire à la fois pour entreprendre l'apprentissage du métier mais aussi pour le mener à bien.

La complexité de l'acte tient à la pathologie et à l'anatomie du patient, au fait que même si l'on devient très habile, une incertitude demeure sur ce que l'on trouvera «en ouvrant» ainsi que sur les suites de l'intervention. L'autre difficulté tient à la capacité de parvenir à la maîtrise fondamentale des gestes nécessaires dans des contextes qui peuvent perturber l'intervention (morphologie du patient, problèmes personnels du chirurgien, articulation avec le travail des autres intervenants, changement des habitudes, etc.).

Les jeunes apprennent par étapes à réaliser des interventions de plus en plus complexes et à être progressivement autonomes pour les mener. Ainsi le chirurgien tient la main au sens propre de l'étudiant, le guide, l'accompagne, accepte à une certaine étape de l'apprentissage qu'il devienne plus autonome. C'est le professeur qui en dernier lieu délivre à l'étudiant la reconnaissance dont il a besoin pour faire en autonomie progressive les bons gestes. Ces apprentissages maître-élève empruntent des caractéristiques communes à celles du compagnonnage. L'élève doit préalablement être adoubé par le maître. Il se confronte aux épreuves de la pratique, acquiert les gestes. La sûreté du geste n'est pas uniquement liée au fait que l'on sait faire. Elle est aussi liée à la confiance que les chirurgiens ont dans leurs capacités. Cette confiance s'acquiert dans l'entraînement, la répétition du geste, mais aussi grâce à la relation positive qui se construit avec l'enseignant. Le compagnonnage est la seule forme d'apprentissage en chirurgie et repose sur un lien fort entre maitre et élève.

Dans la mesure où la chirurgie est un milieu souvent hostile pour les femmes, en particulier dans les modalités d'accueil réservées aux étudiantes, la construction de ce lien maître-élève est problématique. Les blagues de carabin fusent (GODEAU, 2007), mettent parfois mal à l'aise les jeunes femmes et marquent une certaine distance entre leurs références et celles de ce milieu dans lequel elles veulent s'intégrer. L'hostilité à leur égard se manifeste aussi dans les comportements sexistes, les remarques désobligeantes répétées, les apostrophes et petits mots dont elles font l'objet : hostile, stigmatisant et infantilisant, le sexisme ambiant est tout à la fois lassant et mal supporté par les étudiantes comme en témoigne l'extrait d'entretien ci-dessous :

«La chirurgie a un côté positif satisfaisant. On ouvre, on voit, on touche, c'est le bon côté : on voit l'anatomie. La contrepartie est une ambiance épouvantable. Les chirurgiens individuellement peuvent être très sympas mais pas avec les externes. Ils font énormément de blagues machistes, salaces. Même ceux qui s'investissent énormément dans le domaine scolaire et forment les externes; ils peuvent être excessivement méprisants. Si c'est une fille, ça tombe encore plus sur elle...»

(Externe, $2^{\mathrm{e}}$ année, femme, 22 ans.)

Les modalités de l'accueil sont déterminantes dans la motivation pour devenir chirurgien. Presque tous nos interviewés - internes ou chirurgiens en poste - évoquent la figure d'un-e professeur-e qui leur a donné envie de prendre pied dans une spécialité. Nous avons recueilli peu de récits d'externes mais ils sont très tranchés, très hostiles. Les descriptions sont proches de celles faites par les internes, hommes et femmes, à la seule différence que les internes acceptent ces violences comme une caractéristique intangible qu'ils sont obligés d'accepter. Leurs propos sont également corroborés par des représentants du syndicat des internes que nous avons rencontrés et par les chirurgiens viscéraux eux-mêmes qui revendiquent ces pratiques dont l'objectif est soit d'endurcir les aspirants chirurgiens, soit de les aider à choisir ceux qui seront chirurgiens. Il s'agit de fait d'une sorte de sélection par «épreuves psychiques». Une telle violence dans les relations est corroborée par d'autres travaux (Zolesio, 2009, 2013, article dans ce numéro).

Difficile pour les jeunes externes de toujours se mettre à distance et de se protéger d'autant que les remarques portent non seulement sur les compétences en cours d'acquisition mais aussi sur la personnalité. Les jugements lapidaires à vocation prédictive se succèdent: "Vous serez un mauvais médecin». Nombreux sont ceux qui, hommes ou femmes, n'osent pas répondre lorsqu'on les insulte et pleurent tous les jours. Un externe raconte : "Lorsqu'on ne sait pas, ils n'expliquent pas, ils insultent» (externe, $2^{\mathrm{e}}$ année, homme, 23 ans).

Or, le statut de professeur et la reconnaissance médicale dont les $\mathrm{PU}(-\mathrm{PH})$ peuvent se prévaloir donnent légitimité et portée à leurs propos. Dans le même temps, la nouveauté des situations rencontrées par les étudiants et l'étendue des savoirs qu'ils ont à acquérir s'ajoutent à ce qui apparaît ici comme des éléments de vulnérabilité, à savoir la jeunesse et le genre des étudiantes: 
«Si un externe ne sert pas bien pendant une opération, par exemple, [s'il] ne repère pas immédiatement des outils réclamés, cela va mal se passer. Les apostrophes sont machistes du type "viens ici te faire fouetter le gland", "quelle conne", "grosse merde". Ils ne supportent pas qu'il y ait autant de filles qui réussissent et qui soient présentes dans les stages. Sans aucun doute, la présence de dix filles sur dix externes est difficilement acceptée par les chirurgiens. Les filles réussissent mieux que les garçons. Dans ma promo, on est $75 \%$ de filles. On n'a pas envie de se faire traiter comme ça.»

(Externe, 2e année, femme, 22 ans.)

Ce mauvais accueil réservé aux étudiantes mêle deux dimensions qui participent du brouillage des différences entre les sexes. La première est relative au piètre accueil que tout service réserve à l'ensemble des étudiants. La seconde concerne spécifiquement les femmes déconsidérées du fait de leur sexe. Nos observations confirment ainsi les analyses de ZolEsio $(2014,2013)$ qui précise que la domination masculine est constante et ouvertement affirmée et assumée en chirurgie viscérale.

Certaines des externes que nous avons rencontrées nous ont dit ne pas vouloir entrer dans ce milieu qui ne leur reconnaît même pas le droit d'y exister. Après six ans d'études, le défi de devoir démontrer qu'elles ont le droit d'avoir une place en tant que femmes dans la vie professionnelle ne les intéresse pas. Elles refusent de travailler dans une enclave rétrograde.

\section{Mal-être au travail dans un monde sexiste}

Les futures chirurgiennes, tout comme leurs homologues masculins, ont donc à affronter et à apprendre à gérer les différences de valeurs et de fonctionnement entre le milieu - communautaire de la chirurgie et la société dans son ensemble. Nous l'avons vu, le malaise vient également du fait qu'en chirurgie, les femmes sont déconsidérées et stigmatisées alors que dans le même temps, certains chirurgiens se sont érigés en parangons de la toutepuissance et de la virilité. Un tel contexte conduit à l'adoption d'une culture et de comportements propres au milieu, en décalage à la fois avec les modes de socialisation de la majorité des femmes et avec le fonctionnement social actuel. C'est dans ce cadre que les étudiants doivent montrer qu'ils gagnent en expérience et en savoir-faire mais aussi en compétences relationnelles. Insultés, vilipendés, soumis par leurs professeurs à des épreuves relationnelles répétitives, pour certains, à la limite de ce qu'ils peuvent accepter ou plutôt supporter, ils peuvent plier ou pleurer mais doivent apprendre à ne pas rompre, ce qui constitue un réel apprentissage.

Les femmes ont beau déployer tous les efforts nécessaires pour réussir et accomplir un parcours d'apprentissage d'excellence, la domination des hommes n'a de cesse de les frapper de plein fouet, comme en témoigne l'extrait d'entretien suivant:
«Vers la fin de sa formation, une collègue ${ }^{(11)}$ se trouve face au chirurgien qui préside le jury et il lui dit: "La place des femmes est à la maison" " (chirurgie infantile, PU, femme, 55 ans). Typiquement ici, le chirurgien chargé de la présidence du jury utilise le pouvoir qui lui est conféré de manière abusive en déniant l'identité sociale objectivement acquise par la candidate: en une phrase assassine et en une seule confrontation, il balaie des années de construction patiente des savoirs et d'efforts pour atteindre un poste hautement qualifié en renvoyant la candidate à sa représentation sexiste du milieu chirurgical. La violence du propos et son caractère éminemment sexiste apparaissent d'autant plus importants qu'ils concernent une personne brillante, qui a réussi deux concours, dont celui de l'internat ${ }^{(12)}$, en étant très bien classée et après treize ans d'études. Un tel exemple est emblématique des difficultés que les femmes doivent affronter tout au long de leur parcours, y compris parfois jusqu'au jour de la cérémonie, symbolique, de l'attribution $\mathrm{du}$ diplôme. Toutes les femmes que nous avons rencontrées soulignent le sexisme des chirurgiens et décrivent la chirurgie viscérale et orthopédique comme un milieu très hiérarchisé. L'hostilité à l'égard des femmes va très loin puisque même leurs particularités biologiques sont stigmatisées : «Une de mes collègues était enceinte; le chef de service lui a dit en l'apprenant: "Toutes mes condoléances"” (gynécologue, maître de conférences, femme, 45 ans $\left.{ }^{(13)}\right)$. Il n'est d'ailleurs pas rare que certains hommes remettent en question la volonté d'une femme de faire réellement chirurgie et tentent de l'en dissuader au motif qu'elle arrêtera d'exercer à la naissance de ses enfants.

Les femmes disent ressentir dans ces contextes, une absence de respect de la personne, un abus de la position de supériorité conférée par les savoirs et le statut. Quelques externes que nous avions interrogées en 2011 nous décrivaient la mauvaise ambiance des blocs, le rejet dont elles étaient l'objet, le fait que nombre d'entre elles étaient harcelées parfois de manière extrême, jusqu'à la crise de larmes. Certains professeurs eux-mêmes nous ont présenté les violences verbales comme des formes de bizutage, des rites initiatiques, visant à éprouver et forger le caractère des étudiants. Ces relations «laissent des traces» et ce, d'autant plus que les jeunes ne sont pas en position de riposter. Ainsi, les internes femmes ont à affronter une double épreuve. Elles accomplissent un travail pour se former et acquérir les savoirs qui fonderont leur légitimité professionnelle. Elles doivent aussi subir des stigmatisations.

Plus dévastatrices que formatrices, ces relations de domination mettent en évidence le fait qu'une des

(11) Les faits datent de 2006.

(12) L'internat était alors un concours.

(13) Voir aussi Zolesio (2009a). 
qualités requises pour se maintenir dans le milieu est une forte personnalité avec une capacité de mettre les «autrui de référence» à distance. Un monde «dur» $\mathrm{du}$ fait des conditions d'exercice du métier. Toutes les chirurgies n'ont pas la même réputation. Ainsi, "l'orthopédie, ce sont des carabins, très sexistes, héritage de l'armée, ambiance caserne» (chirurgie ophtalmologique, chef de clinique, homme, 55 ans). Ces attitudes sont moins courantes dans les services où les femmes en poste sont plus nombreuses. La violence varie d'un établissement à l'autre; ainsi, c'est dans les établissements les plus prestigieux (à Paris par exemple), qui sont aussi les plus demandés pour les stages d'interne et pour lesquels la concurrence est forte, qu'elle est le plus intense.

Précisons enfin que les femmes n'ont pas d'alternative: elles doivent absolument obtenir la possibilité d'intervenir sur le malade avec l'aide du chirurgien senior. Certaines, craignant de ne pouvoir le faire, s'angoissent beaucoup. L'enjeu est pour elles tellement capital qu'il relègue au second plan les relations délétères avec les chirurgiens seniors d'autant qu'elles savent qu'elles doivent en passer par là et que ce n'est que pour le temps, limité, de leur apprentissage, à l'issue duquel elles pourront intégrer des spécialités avec un autre ethos.

Il reste à comprendre comment les femmes entrées dans une spécialisation chirurgicale résistent dans une telle ambiance.

\section{Le jeu entre identité pour soi et identité pour autrui : les modalités de l'accommodation}

Selon CAssel, les femmes doivent se conformer aux attentes sociales sexuées. Zolesio (2009a, $2009 b$ ) pointe de son côté le fait que les chirurgiennes suivent un parcours de masculinisation qui les conduit à gommer certains stéréotypes de la féminité et à intégrer et manifester, en fonction de leur trajectoire antérieure, des dispositions masculines qui sont fortement ancrées dans la culture des chirurgiens (BERCOT et al., 2011). Cette socialisation professionnelle est toutefois en partie réversible puisque des étapes de "re-féminisation» relative sont observables une fois passée l'étape de l'internat (Zolesio, 2009a).

Entrées malgré un contexte peu accueillant et qui véhicule une représentation stéréotypée des rôles sociaux liés au genre, les femmes déploient des stratégies différenciées et mettent en œuvre des interactions et postures mentales différentes pour s'intégrer. Rappelons que l'enjeu principal est de se faire «adouber» afin d'être conseillée et accompagnée, pour pouvoir tenir les instruments et réaliser soi-même les interventions : tel est le véritable défi et la priorité que se donnent ces étudiantes. On peut repérer trois types d'attitudes parmi les femmes concernées.

\section{S'identifier aux valeurs masculines et les revendiquer}

Entrer dans le monde de la chirurgie, on l'a vu, implique de tisser des liens de complicité et de se faire accepter par une communauté fondée sur la ressemblance. Par conséquent, certaines femmes cherchent à ne jamais être en reste, disputent parfois sans problème sur le même terrain que les hommes et en rajoutent pour sembler au diapason. Élément de construction de proximité et d'appartenance à une communauté, la plaisanterie à caractère sexué est parfois aussi un moyen de mise à distance et de retournement de la relation. Certaines jeunes femmes prennent les apostrophes et les invectives comme un jeu auquel elles participent, ce qui leur permet de ne pas perdre la face et d'être dans le ton. Si certaines le font volontiers signalant qu'elles sont habituées à vivre avec des garçons, d'autres s'y sentent obligées pour ne pas être l'objet passif des quolibets et pouvoir ainsi s'intégrer: elles ne prennent pas ces plaisanteries comme une attaque personnelle mais comme le mode d'expression d'un sous-monde masculin particulièrement machiste.

Les salles d'opérations sont un microcosme où traditionnellement les hommes dirigent, les femmes infirmières obéissent, et où tout le monde est confiné dans un petit espace pendant plusieurs heures. Les femmes chirurgiennes doivent se fondre dans ce milieu machiste et trouver un équilibre entre ce qu'elles sont et ce que l'on voudrait qu'elles soient, ce qui n'est pas sans contradiction : être féminine et se comporter comme un homme chirurgien. Or elles veulent avant tout rester des femmes, même si plusieurs disent avoir du plaisir à être dans un milieu d'hommes et aimer les plaisanteries de salle de garde : "Je suis pas un mec quand même, même si je fais un métier de mec; je ne suis pas un mec» (chirurgie réparatrice, libéral, femme, 60 ans).

De même, une chirurgienne viscérale disant aimer ce milieu d'hommes, se présente comme très féminine :

«On me reproche beaucoup d'avoir des rapports de séduction parce que je reste féminine, je mets des bijoux, je mets des chaussures à talons même à l'hôpital, que je rentre dans ces plaisanteries et qu'on sait que je suis célibataire et du coup, ça me crée parfois des petits soucis.»

(Chirurgie viscérale, interne, femme, 25 ans.)

Cependant, au cours de l'entretien, la hauteur de ses talons exceptée, son comportement nous a plutôt fait penser à celui d'un homme. Dans ses propos, elle joue tout à la fois de sa féminité et de l'image héroïsée du chirurgien qu'elle présente en creux comme une sorte de Batman se levant la nuit pour faire des greffes et travaillant dans l'urgence.

Les attentes des hommes ne sont pas aussi claires puisqu'on attend des femmes qu'elles aient un comportement semblable à celui de leurs confrères ; 
cependant il arrive qu'on le leur reproche. En fait, quand elles se comportent comme les hommes qui les entourent, en étant à l'aise avec leurs plaisanteries, en en rajoutant même, elles les choquent au risque de voir la suite de leur carrière en pâtir. Adopter la même attitude «libérée» que celle des hommes peut aussi entraîner un rejet professionnel :

«J'ai l'exemple d'une collègue qui était réputée pour être très séductrice et très efficace. Ça s'est très mal passé pour elle, elle l'a payé le prix très fort [sic]. [...] De la part des hommes qui sont, pour l'instant en tout cas, en urologie, les seuls décisionnaires, où sur le moment pendant l'internat, ça a profité à tout le monde, tout le monde en a profité(14) et puis le jour où il a fallu lui donner un poste, ça a été non.»

(Interne, urologie, femme, 25 ans.)

Enfin, dans la mesure où les rapports entre chirurgiens et infirmières sont en grande partie fondés sur l'autorité et la séduction, les apprenties chirurgiennes doivent, quant à elles, instaurer des relations d'un autre type avec le personnel infirmier; une interne nous explique ainsi :

"Le mythe du chirurgien est présent. Les infirmières papillonnent autour du chirurgien. Avec un homme, elles sont dans la séduction. Avec une femme, elles se mettent dans l'affrontement. Je suis dans le respect et la coopération.»

(Interne, chirurgie vasculaire, femme, 26 ans.)

Les chirurgiennes parlent de complicité et de respect mutuel, une attitude générale que l'une d'entre elles résume comme suit: «Les infirmières travaillent avec nous, pas pour nous» (interne, chirurgie viscérale, 25 ans). Cette remarque rappelle l'analyse de CASSELL (2000) qui a mis en évidence des comportements différents des infirmières selon qu'elles travaillent avec des chirurgiens ou des chirurgiennes.

\section{Réduire l'impact du regard prédictif d'autrui et l'ignorer}

Une autre posture consiste à tenir à distance les invectives en les ignorant afin d'en réduire les effets. Les femmes qui pratiquent cette stratégie d'évitement désamorcent ces moments d'affrontement et les banalisent en les considérant comme inévitables dans le milieu qu'elles cherchent à intégrer : «De toute façon c'est le milieu qui veut ça» (interne, chirurgie viscérale femme, 26 ans). Il serait donc vain et coûteux de résister en permanence. Elles ont tellement l'habitude d'être agressées et dévalorisées que certaines n'ont recommencé à y prêter attention que parce que des internes hommes leur en ont fait la remarque. Certes, elles souhaiteraient réagir, mais elles expliquent qu'il leur est difficile de le faire au moment où l'incident se produit car il a lieu

(14) Le terme "profité» désigne ici le flirt et les relations sexuelles. le plus souvent en public et dans des circonstances où la priorité est donnée au malade. Comme nous l'avons déjà mis en avant plus haut, elles préfèrent se concentrer sur ce qui est le plus important: se faire une place dans la relation d'apprentissage. Le positionnement de ce sous-groupe fait parfaitement écho au titre de l'un des chapitres de l'ouvrage de Nicole-Claude Mathieu (1991), L'anatomie politique. Catégorisations et idéologies du sexe: «Quand céder n'est pas consentir».

\section{Éviter de couler}

Enfin, certaines femmes internes font état d'un réel malaise, voire d'une souffrance profonde. Elles ne parviennent pas vraiment à s'imposer dans le milieu chirurgical et font figure de victimes ne réussissant pas à surmonter les blessures infligées par les comportements agressifs et misogynes qu'elles subissent de manière répétée. Et ce n'est sans doute pas un hasard si les femmes qui n'ont pas pu se construire les défenses nécessaires et prendre de la distance par rapport aux comportements des professeurs hommes sont davantage issues de milieux sociaux populaires.

Leur position est fragile. Tendues à l'extrême, épuisées, elles envisagent de renoncer à la formation les jours de grand découragement. Elles sont au bord de la rupture mais l'importance du chemin parcouru rend l'idée d'interruption des études inacceptable. Pour éviter de couler, elles se nourrissent de l'appui d'autres étudiants avec lesquels elles partagent leurs difficultés. Ce ne sont pas des conseils concernant la manière de s'y prendre dans leurs relations avec les professeurs qu'elles recherchent mais plutôt une écoute et une solidarité qui les réconfortent. L'enjeu ici comme dans d'autres situations de harcèlement moral est de parvenir à se persuader qu'elles ne sont pas seules à être concernées par ces relations dégradées mais que la situation est liée à un contexte plus général de domination.

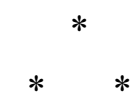

Des travaux ont montré que l'évitement de certains métiers «masculins» correspond de la part des femmes à une volonté de pouvoir mieux gérer l'interface famille-travail (CROMPTON, LEFEUVRE, 1997). Au terme de notre enquête, il apparaît que les femmes qui ont choisi la médecine ont souvent été encouragées par leurs familles. Néanmoins, lorsqu'elles choisissent la chirurgie, leur entourage amical tente souvent de les en dissuader, en invoquant l'incompatibilité de la durée du travail en chirurgie avec une vie de famille. En effet, la durée du travail y a été organisée, nous l'avons vu, en lien avec un ethos essentiellement masculin. 
Dans l'univers professionnel de la chirurgie comme dans d'autres, les femmes font les frais d'une stratégie d'exclusion de la part des hommes (Crompton et al., 1999). Nos enquêtes dans des hôpitaux auprès d'internes et de professeurs d'université et/ou hospitaliers ont mis en évidence que les causes y sont aussi socio-historiques. L'apprentissage par compagnonnage allié à un ethos professionnel viril peu favorable à la mixité équivaut à une période de sélection drastique par découragement, et agit comme un repoussoir pour les femmes. Ces différentes dimensions - ethos, hiérarchie et acquisition des savoirs par compagnonnage - forment un système de socialisation qui, tel qu'il est conçu, rend difficile l'intégration des femmes en chirurgie.

Celles qui ont choisi malgré tout la chirurgie sont très investies, à la fois dans les apprentissages et dans le travail qu'elles ont à effectuer dans ce cadre. Elles aspirent à se réaliser et tiennent le cap : acquérir les savoirs d'un métier difficile, à forte responsabilité et demandant un long apprentissage au niveau élevé et aux enjeux relationnels déterminants. Dans la mesure où cette formation passe par le compagnonnage, les femmes doivent non seulement être tolérées mais également acceptées par les seniors. Dans un milieu qui leur est a priori peu favorable, elles doivent parvenir à des interactions réussies avec leur «maître»; pour cela, elles accomplissent un important travail sur elles-mêmes, dont l'un des objectifs est de dépasser les décalages entre identité pour autrui et identité pour soi.

Le monde chirurgical n'est toutefois pas uniforme: progressivement, différentes cultures tendent à coexister, notamment sous l'impact de la féminisation de certaines spécialités; des espaces où l'accueil des femmes est plus favorable et la domination masculine moins prégnante sont ainsi créés. Il n'en demeure pas moins que les premiers pas en chirurgie constituent une véritable épreuve.

\section{Bibliographie}

Bercot R. (2009), «Rationaliser la gestion des blocs opératoires: la négociation des territoires et des légitimités professionnelles», Sciences de la société, $\mathrm{n}^{\mathrm{0}} 76$, pp. 54-65.

Bercot R., Mathieu-Fritz A. (2007), «La crise de recrutement des chirurgiens français : entre mythes et réalités », Revue française de sociologie, vol. 48, no 4 , pp. 751-779.

Bercot R., Horellou-Lafarge C., Mathieu-Fritz A. (2011), «Les transformations récentes de la chirurgie française. Spécialisation, féminisation, segmentation», Revue française des affaires sociales, $\mathrm{n}^{\circ}$ 2-3, pp. 104-122.

Billaut A. (2005), «Les affectations en troisième cycle des études médicales en 2004 suite aux épreuves classantes nationales (ECN)», Études et résultats, no 429, Direction de la recherche, des études, de l'évaluation et des statistiques (Drees).

Bosk C. L. (1979), Forgive and remember. Managing medical failure, Chicago, University of Chicago Press.

Bourdieu P. (1982), «Les rites comme actes d'institution", Actes de la recherche en sciences sociales, no 43 , pp. 58-63.

Cassell J. (2000), «Différence par corps: les chirurgiennes », Cahiers du genre, no 29, pp. 53- 81.

Commaille J. (1993), Les stratégies des femmes : travail, famille et politique, Paris, La Découverte.

Cromer S., Lemaire D. (2007), «L'affrontement des sexes en milieu de travail non mixte, observatoire du système de genre», Cahiers du genre, no ${ }^{\circ} 42$, pp. 61-78.
Crompton R., Le Feuvre N. (1997), «Choisir une carrière, faire carrière en France et en Grande-Bretagne », Cahiers du Genre, no 19, pp. 49-75.

Crompton R., Le Feuvre N., Birkelund G. (1999), " The restructuring of gender relations in the medical profession ", in Crompton R. (ed.), Restructuring gender relations and employment: the decline of the male breadwinner, Oxford, Oxford University Press, pp. 179-200.

Fauvet L. (2010), «Les affectations des étudiants en médecine à l'issue des épreuves classantes nationales en 2009», Études et résultats, no 720, Drees.

Fauvet L., Romain O. (collab.), Buisine S. (collab.), LAURENT P. (collab.) (2012), «Les affectations des étudiants en médecine à l'issue des épreuves classantes nationales en $2011 »$, Études et résultats, n 802 , Drees, Centre national de gestion (CNG).

Godeau E. (2007), L'«esprit de corps » : sexe et mort dans la formation des internes en médecine, Paris, Éditions de la Maison des sciences de l'homme.

Godefroy P., Romain O. (collab.), Buisine S. (collab.), LAurent P. (collab.) (2013), «Les affectations des étudiants en médecine à l'issue des épreuves classantes nationales en 2012», Études et résultats, no 852, Drees, CNG.

Hardy-Dubernet A.-C. (2005), «Femmes en médecine : vers un nouveau partage des professions ?», Revue française des affaires sociales, $\mathrm{n}^{\circ} 1$, pp. 35-38.

HérITIER F. (1996), Masculin, féminin. La pensée de la différence, Paris, Odile Jacob. 
Hughes E. C. (1996), Le regard sociologique: essais choisis, textes rassemblés et présentés par J.-M. Chapoulie, Paris, École des hautes études en sciences sociales.

JAISSON M. (2002), «La mort aurait-elle mauvais genre? La structure des spécialités médicales à l'épreuve de la morphologie sociale», Actes de la recherche en sciences sociales, no 143 , p. 44-52.

Katz P. (1999), The scalpel's edge. The culture of surgeons, Needham Heights, Allyn and Bacon.

Lapeyre N., Le Feuvre N. (2005), «Féminisation du corps médical et dynamiques professionnelles dans le champ de la santé», Revue française des affaires sociales, $\mathrm{n}^{\circ} 1$, pp. 59-81.

Mathieu N.-C. (1991), L'anatomie politique: catégorisations et idéologies du sexe, Paris, Côté-femmes.

Piaget J. (1965), Études sociologiques, Genève, Droz.

Pruvost G. (2007), Profession, policier : sexe, féminin, Paris, Éd. de la Maison des sciences de l'homme.

ROCHER G. (1968), Introduction à la sociologie générale. Tome 1 : L'action sociale, Paris, Seuil.

SiCART D. (2005), «Les médecins. Estimations au $1^{\mathrm{er}}$ janvier $2005 »$, Document de travail, Série Statistiques, n 88, Drees.
Simmel G. (1999), Sociologie. Études sur les formes de la socialisation, trad. de l'allemand par L. Deroche-Gurcel et S. Muller, Paris, Presses universitaires de France.

TönNies F. (1977 [1 $1^{\text {re }}$ éd. 1922]), Communauté et société. Catégories fondamentales de la sociologie pure, Introduction et traduction de J. Leif, Paris, Presses universitaires de France.

Zolesio E. (2009a), «Marie Laborie, un cas de socialisation chirurgicale ratée», Sociétés contemporaines, no 74 , pp. 147-165.

Zolesio E. (2009b), «Des femmes dans un métier d'hommes: l'apprentissage de la chirurgie», Travail, genres et sociétés, no 22, pp. 117-133.

Zolesio E. (2011), «De la nécessité pour les femmes chirurgiens de "faire leurs preuves"», Questions vives [en ligne], vol. $8, \mathrm{n}^{\circ} 15$; disponible en ligne à l'adresse : http:// questionsvives.revues.org/741; consulté le 24 février 2015.

Zolesio E. (2012), Chirurgiens au féminin? Des femmes dans un métier d'hommes, Rennes, Presses universitaires de Rennes.

Zolesio E. (2013), «La socialisation chirurgicale, un apprentissage "par claques"», Revue française de pédagogie, no 184, pp. 95-104. 